\title{
Isotopic Fractionation in Slow and Coronal Hole Associated Solar Wind
}

\author{
H. Kucharek, B. Klecker
}

Max-Planck-Institut für extraterrestrische Physik, P.O. Box 1312, 85741 Garching, Germany

F. M. Ipavich

University of Maryland, College Park, USA

R. Kallenbach

International Space Science Institute, Bern, Switzerland

H. Grünwaldt

Max-Planck-Institut für Aeronomie, Katlenburg-Lindau, Germany

M. R. Aellig

MIT/Center for Space Research, Cambridge, USA

P. Bochsler

Physikalisches Institut, Universität Bern, Bern, Switzerland

\begin{abstract}
Data from the Mass and Charge Time-of-Flight spectrometers MTOF and CTOF on board SOHO have been accumulated for time periods in which coronal hole and non-coronal hole type plasma has been detected by using different methods in order to determine the abundance ratios of magnesium isotopes in these two different source regions of the solar wind. As indicators for the two different solar wind type plasma we have used several plasma parameters such as, the freeze-in temperatures, the $\mathrm{Fe} / \mathrm{O}$ and the $\mathrm{He} / \mathrm{H}$ ratio, which are known as reasonable indicators. For this study we have used the refractory element $\mathrm{Mg}$ which is an authentic whitness for matter in the solar nebula because temperatures of the Sun never have been high enough to change their isotopic composition by nuclear burning. In addition $\mathrm{Mg}$ has three isotopes with abundance ratios greater than about $10 \%$ and therefore they are easy to observe.
\end{abstract}

\section{Introduction}

The study of the solar isotopic composition is motivated by the interest in the composition of the early solar nebula which provides knowledge about the history of the solar system. It also provides the opportunity to analyze solar matter 
in situ and get information about the present day composition of the outer convective zone of the Sun. The flow dynamic of the solar wind is determined by the magnetic topology of the flux tubes. The slow solar wind originating from closed field line regions, has a slow bulk velocity and high freeze-in temperature. The high speed solar wind has a low freeze-in temperature and originates from open flux tubes in coronal holes. Recent studies (Bochsler et al., 1997, Kucharek et al., 1998, Wimmer et al. 1998) have shown that the isotopic compositions of the refractory elements are close to their solar wind compositions. The question appears how well these elements represent the solar source composition and whether there is a difference in the isotopic composition of refractory elements in the fast and slow solar wind type plasma. Small variations of the isotopic abundance ratios of the refractory elements can be discussed in the context of experimental (von Steiger et al., 1995) and theoretical (Bodmer and Bochsler,1996) studies on Coulomb friction in the solar wind plasma.

\section{Sensors, data selection and results}

In order to investigate possible fractionation processes in the solar wind data from the high resolution Mass Time-Of-Flight spectrometer MTOF and the Charge Time-Of-Flight spectrometer CTOF on board SOHO have been used. In addition we have used data from the WIND/SWE experiment on board the WIND space craft. Since details of all these sensors and details of calibration and data format can be found elsewhere we concentrate on the different methods for data filtering only. In this paper we will report on three different methods to identify slow or coronal hole associated solar wind. The so-called freeze-in temperatures are a very good tool for coronal diagnostic. These quantities are derived by assuming that there is an ionization equilibrium in the corona and applying ionization and recombination rates for colliding electrons and ions. They are established a few solar radii above the solar surface and they do not change on their way throughout the interplanetary medium. This is in contrast to the dynamic properties of the solar wind, like the solar wind speed or solar wind density. Usually coronal hole solar wind is associated with a freeze-in temperatures of 1.2-1.3 MK and slow solar wind with 1.5-2.0 MK. Another important parameter to distinguish between these two classes of solar wind is the $\mathrm{Fe} / \mathrm{O}$ ratio. In the solar wind, elements with low First Ionization Potential (FIP) $(<10 \mathrm{eV})$ are systematically enriched relative to high FIP elements compared to their photospheric abundance. The enrichment factor ranges from 3-5 for slow solar wind associated with the streamer belt, and is about two for fast solar wind associated with coronal holes (v. Steiger et al. (1995)). Oxygen is the most prominent high FIP element whereas Fe is one of the most prominent low FIP elements. From previous investigations (Aellig et al., 1998), (Ipavich et al., 1992), (Geiss et al., 1995) it is known that the Fe/O ratio shows two distinct regions which associated with slow $(\mathrm{Fe} / \mathrm{O} \sim 0.12)$ and fast solar wind $(\mathrm{Fe} / \mathrm{O} \sim 0.06)$. One of the largest variations in the solar wind composition is the depletion of ${ }^{4} \mathrm{He}^{2+}$ in the slow solar wind compared to its abundance in the fast coronal-hole type solar wind. The depletion of ${ }^{4} \mathrm{He}^{2+}$ is associated with the sector boundaries which are coronal signatures of the slow interstream solar wind. Due to the least favorable drag factor of all minor solar wind ions they 
must be depleted. For this study we have used the abundance ratios of $\mathrm{He} / \mathrm{H}$ from WIND/SWE experiment. We have associated a ratio of $\mathrm{He} / \mathrm{H} \sim 0.02$ with slow and a ratio of $\mathrm{He} / \mathrm{H} \sim 0.03$ with fast solar wind. According to the work of Bodmer and Bochsler (1996) the fractionation of ${ }^{24} \mathrm{Mg}$ and ${ }^{26} \mathrm{Mg}$ is larger in the slow solar wind than in the fast solar wind in the sense that heavier isotopes with their less favorable drag factors are depleted in the slow solar wind. The MTOF sensor on board SOHO is an isochronous Time Of Flight mass spectrometer which provides the possibility to resolve the different isotopes of almost all solar wind elements between 3 to $60 \mathrm{amu}$. For the results presented below we have filtered the mass spectrograms by using the above mentioned parameters as a filter for coronal hole and slow solar wind type plasma. In addition the spectra have been corrected with respect to instrumental isotope fractionation. For details of the data filtering we would like to refer to much more detailed papers (see Kallenbach et al., 1997, Kucharek et al., 1998). The table below shows the abundance ratio of ${ }^{24} \mathrm{Mg} /{ }^{26} \mathrm{Mg}$ (- the mass dependend effect is most prominent in this case -) for slow solar wind and coronal hole associated solar wind by using the different filtering methods.

Table 1. $\quad{ }^{24} \mathrm{Mg} /{ }^{26} \mathrm{Mg}$ abundance ratio for slow, coronal hole associated solar wind and terrestrial value.

\begin{tabular}{rccc}
${ }^{24} M g /{ }^{26} M g$ & $\mathrm{Fe} / \mathrm{O}$ & Freeze-in & $\mathrm{He} / \mathrm{H}$ \\
\hline slow sw & $7.7 \pm 0.12$ & $7.9 \pm 0.13$ & $7.55 \pm 0.15$ \\
coronal hole & $7.2 \pm 0.14$ & $7.1 \pm 0.15$ & $7.11 \pm 0.16$ \\
terrestrial & 7.17 & 7.17 & 7.17 \\
\hline
\end{tabular}

\section{Summary}

All results presented in the table indicate that there is a depletion of heavier Magnesium isotopes in the slow solar wind which is in the order of $1.5 \% \pm 1.4 \%$ ( $2 \sigma$ error) per mass unit compared to its abundance ratio in the fast/coronal hole associated solar wind (see also Kallenbach et al., 1998). This is a pure mass dependent effect which indicate isotopic fractionation in the solar wind. The effect of inefficient Coulomb coupling predicts the observed trend.

\section{References}

Aellig, M.R, et al., 1998, JGR, 103, 17215.

Bochsler, P, et al., 1997, Proc of the 8th Int. Solar Wind Conf, 199.

Bodmer, R. et al., 1996, Proc. of the Eur. Geophys. Soc., The Hague.

Geiss, J., et al., 1995, Space Sci. Rev., 72, 49.

Ipavich, F.M., et al., 1992, Solar Wind Seven, p 369.

Kallenbach, R., et al., 1997, JGR, 102, 26895.

Kallenbach, R., et al., 1998, Space Sci. Rev., 85, 357.

Kucharek, H., et al., 1998, JGR, 103, 26805.

v. Steiger, R., et al., 1995, Adv. Space Res., 15, (7)3.

Wimmer, R., et al., 1998, JGR, 103, 20621. 